\title{
Aberrantly expressed miR-188-5p promotes gastric cancer metastasis by activating Wnt/ $\beta$-catenin signaling
}

\author{
Yun Li ${ }^{1,2 \dagger}$, Xiaoli Yan ${ }^{1 \dagger}$, Jiajian Shi ${ }^{1}$, Yun He ${ }^{1}$, Jie Xu', Liying Lin ${ }^{1}$, Wannan Chen ${ }^{3}$, Xinjian Lin ${ }^{1 *}$ and Xu Lin ${ }^{1,3^{*}}$
}

\begin{abstract}
Background: Gastric cancer (GC) is one of the most common human cancers with the high rate of recurrence, metastasis and mortality. Aberrantly expressed microRNAs (miRNAs) are associated with invasion and metastasis in various human cancers. Recently, miR-188-5p has been indicated as an oncogene in GC since it promotes GC cell growth and metastasis. However, the underlying molecular mechanism remains to be fully defined.
\end{abstract}

Methods: Using Significance Analysis of Microarrays (SAM) screening, we identified that miR-188-5p is associated with overall survival and lymph node metastasis in patients with GC. The functional impact of miR-188-5p on GC metastasis was validated using in vitro and in vivo assays. The regulatory function of miR-188-5p on Wnt/ 3 -catenin signaling activation through directly targeting PTEN was proven using quantitative real-time PCR, western blot analysis, a dual-luciferase assay, a Transwell assay, and immunofluorescence. Immunohistochemical analyses further confirmed the clinical significance of miR-188-5p in GC.

Results: MiR-188-5p diminishes tumor suppressor PTEN expression, and further increases phospho-Ser9 of GSK3 $\beta$ to activate Wnt/ $\beta$-catenin signaling in GC. Consequently, miR-188-5p enhanced the migration and invasion of GC cells in vitro and tumor metastasis in vivo, whereas inhibition of miR-188-5p had the opposite effects. Moreover, miR188-5p was negatively correlated with PTEN expression but positively correlated with nuclear $\beta$-catenin staining in GC samples.

Conclusions: Our findings revealed a model of the miR-188-5P-PTEN- $\beta$-catenin axis in GC, which mediates the constitutive activation of Wnt/ $\beta$-catenin signaling and promotes tumor metastasis, inferring that miR-188-5p is a potential therapeutic target to treat GC.

Keywords: MiR-188-5p, Gastric cancer, Metastasis, Wnt/ß-catenin signaling

\section{Background}

Gastric cancer (GC) is characteristic of poor prognosis and high mortality, making it the third most widely diagnosed malignancy worldwide and the cause of $12 \%$ of all cancer-related deaths each year [1]. Tumor metastasis is common and represents a major obstacle to improvements in GC patient survival [2]. Therefore, there is a urgent need to identify new prognostic markers and to

\footnotetext{
* Correspondence: xlin@mail.fjmu.edu.cn; xlin@fjmu.edu.cn; linxu@mail.fjmu.edu.cn

†Yun Li and Xiaoli Yan contributed equally to this work.

${ }^{1}$ Key Laboratory of Gastrointestinal Cancer (Fujian Medical University), Ministry of Education, Fuzhou, China

Full list of author information is available at the end of the article
}

develop novel therapeutic strategies to treat metastatic GC. The evolution of gastric tumorigenesis involves a series of sequential steps. Among these characteristic events of GC metastasis is hyperactivation of $\mathrm{Wnt} / \beta$-catenin signaling. Activated $\mathrm{Wnt} / \beta$-catenin signaling correlates with aggressiveness of GC with poor patient survival [3]. Moreover, a high level of $\beta$-catenin activity, stimulated by abnormal expression of Wnt-5a, functionally enhances GC cells' migratory and invasive abilities [4]. Conversely, ablation of $\beta$-catenin resulted in suppression of metastasis in GC [5].

Wnt $/ \beta$-catenin signaling is triggered upon binding of active Wnt ligands to their membrane receptors frizzled (FZD) [6] and LDL receptor-related protein 5 or 6 
(LRP5/6) [7]. The ligand-receptor interaction can cause dissociation of $\beta$-catenin from the "destruction complex" comprising Axin, APC, CK $1 \alpha$, and GSK3 $\beta$ [8]. As a consequence, increased free cytosolic $\beta$-catenin translocates to the nucleus to induce the transcription of its downstream genes related to tumorigenesis. In the absence of Wnt ligand stimulation, cytosolic $\beta$-catenin is normally sequestrated in the "destruction complex" and Wnt/ $\beta$ catenin signaling is inactivated $[9,10]$. Additionally, crosstalk between Wnt/ $\beta$-catenin signaling and other pathways such as PI3K/Akt pathway has been implicated in numerous cancers [11]. The tumor suppressor gene, phosphatase and tensin homolog (PTEN), is frequently inactivated in various tumor types. In addition, that PI3K/Akt suppression inhibits $\beta$-catenin activity is one of the major mechanisms by which PTEN exerts its suppressive effect on tumor progression $[12,13]$. While signaling in Wnt/ß-catenin pathway is deliberately controlled, its constitutive activation is frequently observed in many human cancer types. Therefore, revealing how these factors regulate the $\mathrm{Wnt} / \beta$-catenin signaling pathway in cancers is of biological and clinical important for the future development of more efficacious therapeutic strategies.

MicroRNAs (miRNAs or miRs) are short non-coding RNAs that bind to 3 '-untranslated regions (UTRs) of the mRNAs of target genes causing their post-transcriptional inhibition or degradation [14]. Besides their crucial roles in cellular differentiation and organism development, miRNAs are frequently misregulated in human cancer $[15,16]$. Distinct miRNAs could act as key regulators of the Wnt/ $\beta$-catenin signaling pathway in a variety of human malignancies including breast [17], liver [18] and colon cancer [19]. However, whether miRNA dysregulation could interfere with important Wnt/ $\beta$-catenin signaling suppressors for aberrant activation of this signaling pathway in GC remains largely unknown.

In the present study, we identified miR-188-5p as an important miRNA that is closely correlated with GC metastasis and poor patient survival. Mechanistically, by inhibiting PTEN expression, miR-188-5p appears to function as the activator of the Wnt/ $/$-catenin pathway leading to an increase in GC cell invasion in vitro and tumor metastasis in vivo. These results suggest that miR-188-5p contributes to promoting GC metastasis, and may represent a novel therapeutic target for the treatment of GC.

\section{Methods}

\section{Cell culture}

A total of seven human GC cell lines (MKN74, AGS, KATOIII, NUGC3, MGC803, MKN45, and HGC27) were purchased from the Type Culture Collection of the Chinese Academy of Sciences (Shanghai, China) and maintained in RPMI-1640 medium (Invitrogen, Carlsbad, CA,
USA) except AGS in Ham's F12 medium (Invitrogen). All media were supplemented with $10 \%(\mathrm{v} / \mathrm{v})$ fetal bovine serum (Invitrogen).

\section{The Cancer genome atlas (TCGA) data analysis} miRNA-seq data from 378 stomach tumor samples with $\mathrm{N}$ stage information were downloaded from the TCGA stomach cancer data set portal (http://tcga-data.nci.nih. gov/tcga accessed on 2016/3/10; TCGA accession codes are listed in Additional file 1: Excel spreadsheet 1. Among these 378 stomach tumor samples, 307 samples also had overall survival (OS) data. Clinical information on the recruited patient specimens is shown in Additional file 2: Table S1. BRB-Array Tools [20] were used to perform the data analysis. The exclusion criteria for a given miRNA were as follows: less than $20 \%$ of the expression data showing at least a 1.5-fold change in either direction from the miRNA's median value, or the percentage of data missing or filtered out exceeding $50 \%$.

\section{Tissue specimens}

GC samples and their corresponding adjacent nontumor tissues were collected and histopathologically diagnosed at the Union Hospital of Fujian Medical University (Fuzhou, China) and relevant clinical information on the recruited patients is presented in Additional file 3: Table S2. These specimens are designated here as the FMU cohort. The patients' informed consent and approvals from the Institutional Research Ethics Committee were obtained.

\section{Significance analysis of microarray data}

Microarray data were analyzed using MeV4.9 (http:// www-stat-class.stanford.edu/SAM/SAMServlet) with the SAM (significance analysis of microarrays) program (Stanford University, Stanford, CA, USA) [21] which identifies the genes most closely correlated with survival time or lymph node metastasis status with simultaneous permutation analysis for estimation of the false discovery rate (FDR).

\section{Gene set enrichment analysis (GSEA)}

Global mRNA expression profiles on the TCGA stomach cancer specimens with the availability of miR-188-5p expression data were subjected to GSEA using GSEA 2.0.9 software (http://www.broadinstitute.org/gsea/) for identifying an association of miR-188-5p with metastasisrelated and $\mathrm{Wnt} / \beta$-catenin signaling pathways, using the methods as previously described [22, 23]. A numeric variable was assigned to miR-188-5p expression and a continuous-type cls file of the miR-188-5p profile was applied to phenotype the labels in GSEA. The metric for ranking genes in GSEA was set as 'Pearson' whereas the other parameters were set to their default values [24]. 


\section{Plasmids and transfection}

A cDNA including the hsa-miR-188 precursor with 500 bp of flanking genomic sequence on each side was cloned into the retroviral transfer plasmid pMSCV-puro (Clontech, Mountain View, CA, USA). The open reading frame (ORF) of PTEN was inserted into the mammalian expression vector pcDNA 3.1 (Invitrogen). The 3'-UTR of PTEN was placed downstream of the luciferase gene in a pmirGLO dual-luciferase miRNA target expression vector (Promega, Madison, WI, USA). The primers used are listed in Additional file 4: Table S3. MiR-188-5p mimic, miR-188-5p inhibitor, and their control oligonucleotides were obtained from GenePharma (Shanghai, China). PTEN-TSB, a customized target site blocker (TSB) to block miR-188-5p binding to PTEN, and non-targeting control TSB LNA oligonucleotides were purchased from Exiqon (Vedbaek, Denmark). Transfection of plasmids or oligonucleotides was performed using X-tremeGENE reagent (Roche Diagnostics, $\mathrm{GmbH}$, Mannheim, Germany) according to the manufacturer's instructions.

\section{RNA extraction and quantitative real-time PCR}

Total miRNA isolation and cDNA synthesis were performed using commercial kits according to the manufacturer's instructions. miRNA expression was quantified with qPCR using miRNA-specific primers in a real-time PCR system as previously described [25]. Additional file 5: Table S4 shows the sequences of the primers used.

\section{Western blot analysis and immunofluorescent assays}

Western blotting and immunofluorescent assays were performed according to corresponding standard methods [25]. The specific antibodies used were listed as follows: PTEN (Cell Signaling Technology (CST), 1:1000 dilution), GSK3 $\beta$ (CST, 1:800 dilution), Akt (CST, 1:800 dilution), phospho (p) Akt (Ser473) (CST, 1:500 dilution), pGSK3ß (Ser9) (CST, 1: 500 dilution), GAPDH (CST, 1:5000 dilution), $\beta$-catenin (Abcam, 1:50 dilution), and Alexa Fluor 594-conjugated goat anti-rabbit secondary antibody (1:200, $2 \mathrm{mg} / \mathrm{mL}$, Invitrogen). Fluorescence images were captured by a laser scanning confocal microscope (Zeiss, Germany).

\section{Cell proliferation assay}

Cells were seeded at a density of $5 \times 10^{3}$ cells per well in 96-well plates and cultured for 24, 48, 72, 96, or $120 \mathrm{~h}$. Cell Counting Kit-8 (CCK-8; Dojindo, Kuma-moto, Japan) was used to evaluate the proliferative capacity of the cells. The absorbance was then measured at the wave length of $450 \mathrm{~nm}$ using a microplate reader (Bio-Tek, Winooski, VT, USA).

\section{Wound-healing assay}

Cells were grown to nearly $100 \%$ confluence on 6-well cell culture dishes and a same size scratch was made through the cell monolayer using a pipette tip. After thorough washing with PBS, fresh culture medium was replenished and the wound closure was photographed at 0,24 and $48 \mathrm{~h}$ to monitor the migration of cells into the wounded area.

\section{Cell migration and invasion assay}

$3 \times 10^{4}$ cells were placed onto the upper chamber of Transwell insert (BD bioscience, San Jose, CA, USA) without or with Matrigel and incubated at $37^{\circ} \mathrm{C}$ for $22 \mathrm{~h}$ followed by removal of cells inside the upper chamber with cotton swabs. The insert was then fixed in $20 \%$ methanol, stained with $0.1 \%$ crystal violet and counted under Qimaging micropublisher 5.0 RTV microscope (Olympus, Tokyo, Japan).

\section{Dual-luciferase reporter assay}

$3.5 \times 10^{4}$ cells were seeded and settled in 24-well plates for $24 \mathrm{~h}$. Indicated plasmids (100 $\mathrm{ng}$ each) plus $1 \mathrm{ng}$ of pRL-TK plasmid (Promega) were transfected into cells. For measurements of $\beta$-catenin transcriptional activity, the reporter plasmid containing the DNA binding site of wild-type (CCTTTGATC; TOP-flash) or mutated (CCTTTGGCC; FOP-flash) TCF/LEF (Addgene, Cambridge, MA, USA) were used. Luciferase and Renilla signals were measured $48 \mathrm{~h}$ after transfection using the Dual-Luciferase Reporter Assay Kit (Promega) according to a protocol provided by the manufacturer.

\section{Tumor xenografts in vivo}

3-million MGC803 cells or 4-million AGS cells stably expressing firefly luciferase and miR-188-5p were injected intravenously via the tail vein of $\mathrm{BALB} / \mathrm{c}$ nude mice. Fifty days after inoculation, the mice were euthanized by carbon dioxide inhalation followed by cervical dislocation and the lungs were removed and examined for any evidence of metastasis by a dissecting microscopy and histopathologic analysis. IVIS Spectrum Imaging System (Caliper Life Sciences, Hopkinton, MA, USA) was employed for a whole-mouse bioluminescence imaging. Living Image 4.2 software (Caliper Life Sciences) was used for image calibration and visualization. The animal study was approved by the Institutional Animal Care and Use Committee of Fujian Medical University.

\section{Immunohistochemistry}

Immunohistochemistry was performed according to the standard streptavidin-biotin- peroxidase complex method. After sections were stained using anti-PTEN (CST, 1:100 dilution) and anti- $\beta$-catenin (CST, 1:100 
dilution), images were captured using the AxioVision Rel.4.6 computerized image analysis system (Carl Zeiss). The degree of immunostaining of indicated proteins was evaluated and scored as previously described [25].

\section{Statistical analysis}

All statistical analyses were carried out using the SPSS 20.0 statistical software package except for the microarray data. Power analysis was performed to determine sample size in order to achieve a minimum effect size of 0.5 with a $P$ value $<0.05$. The patients were classified into two groups of a high ( $>$ the median) or low $(\leq$ the median) miR-188-5p expression. Overall survival curves were plotted by the Kaplan-Meier method with the logrank test applied to determine a significance. Cox regression was used for analysis of univariate and multivariate survival. The relationship between the expression levels of miR-188-5p and its target genes was analyzed by $\chi^{2}$-test or Fisher's exact test. Student's $t$-test was used to determine differences between groups. All data were represented as mean \pm SD from 3 independent experiments. $P<0.05$ was considered significant.

\section{Results}

miR-188-5p correlates with GC patient survival and lymph node metastasis

To examine whether miRNAs were associated with GC patient survival and lymph node metastasis status, we analyzed the miRNA expression profiles in patients with stomach adenocarcinoma (STAD) using the stomach cancer data set of TCGA. From censored survival analysis of the Significance Analysis of Microarrays (SAM) in MeV4.9, we identified 13 distinctly expressed miRNAs that were closely correlated with OS or lymph node metastasis status, corresponding to a median expected FDR of $10 \%$ (Table 1). Among the 13 miRNAs identified, miR-221-3p and miR-223-3p have been reported of clinical significance in GC [26, 27]. Intriguingly, miR-188-5p appeared to be the only miRNA significantly correlated with both OS and lymph node metastasis status. Moreover, miR-188-5p expression level was much higher than miR-188-3p in the TCGA miRNAHiSeq expression array (Additional file 6: Figure S1A). These findings indicated that miR-188-5p might be potentially more important than miR-188-3p in GC progression.

More specifically, for those STAD patients in the TCGA data set with $\mathrm{N}$ stage data available, the expression of miR-188-5p was markedly higher in stage N1N3, but significantly lower in stage NO STAD samples (unpaired Student's $t$-test, $P<0.01$, Fig. 1a). MiR-188-5p was highly upregulated in lymph node metastatic GC specimens as compared with non-lymph node metastatic samples. Furthermore, as shown in Fig. 1b, the patients with a higher miR-188-5p expression (> median) had a
Table 1 Significance analysis of microarrays (SAM) using the TCGA Stomach Adenocarcinoma (STAD) data set identified microRNAs associated with patients' OS or lymph node metastasis

\begin{tabular}{lc}
\hline microRNAs & $q$-value(\%) \\
\hline High expression correlated with shorter OS & \\
hsa-miR-221-3p & 0.00 \\
hsa-miR-188-5p & 0.00 \\
hsa-miR-223-3p & 0.00 \\
Low expression correlated with shorter OS & \\
hsa-miR-145-5p & 0.00 \\
hsa-miR-29a-3p & 0.00 \\
hsa-miR-200b-3p & 0.00 \\
hsa-miR-194-5p & 3.47 \\
High expression correlated with positive-lymph node metastasis \\
hsa-miR-125a-5p & 0.00 \\
hsa-miR-500a-3p & 0.00 \\
hsa-miR-188-5p & 0.00 \\
Low expression correlated with positive-lymph node metastasis \\
hsa-miR-211-5p & 0.00 \\
hsa-miR-147b & 0.00 \\
hsa-let-7b-5p & 0.00 \\
hsa-miR-150-5p & 0.00 \\
\hline Os overal surva
\end{tabular}

OS overall survival

significantly shorter OS than those expressing a lower level of miR-188-5p ( $\leq$ median). Similar results were seen in the FMU cohort (Fig. 1c and d). Moreover, an additional analysis of miR-188-5p expression between M0 and M1 patients in TCGA STAD cohort also revealed that miR-188-5p was significantly upregulated in M1 patients as compared with M0 patients (unpaired Student's t-test, $P=0.0332$; Additional file 6: Figure $\mathrm{S1B})$, suggesting that miR-188-5p level is correlated with tumor distant metastasis in GC patients.

We then evaluated the expression level of miR-188-5p in tumor specimens and GC cell lines. Figure 1e showed that expression of miR-188-5p was increased in eight randomly selected GC tumor specimens as compared with the paired adjacent non-tumor tissues. The same was true for seven GC cell lines relative to normal gastric epithelial cells (NGECs) (Fig. 1f). To further confirm this, we also analyzed miR-188-5p expression levels in TCGA STAD cohort that demonstrated miR-188-5p was significantly up-regulated in gastric cancers compared to normal tissues $(P<0.0001$; Additional file 6: Figure S1C) . Similarly, analysis of paired adjacent normal and GC tissues revealed that the miR-188-5p level was more than 5-fold increased in GC samples (Additional file 6: Figure S1D and E). Collectively, these data indicated that miR-188-5p was markedly upregulated in GC. 
A

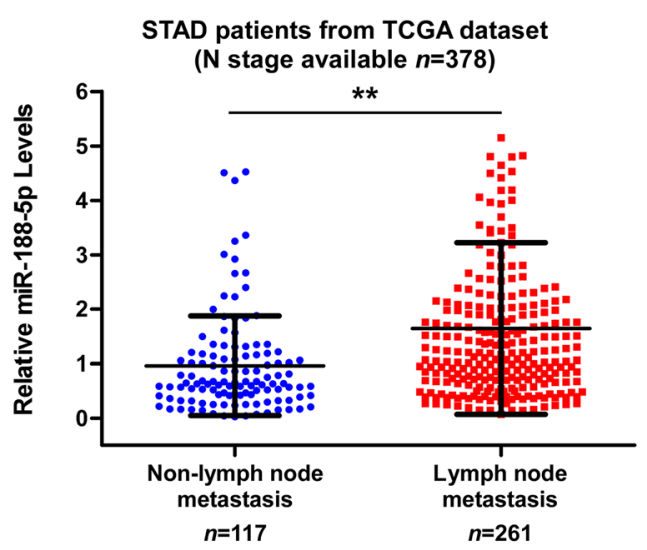

C STAD patients from FMU cohort $(n=116)$

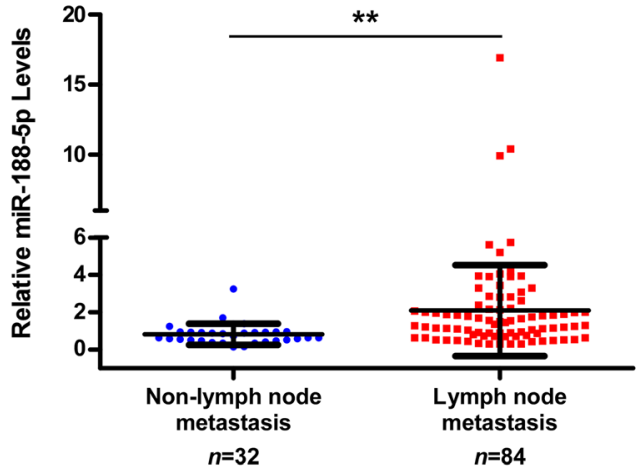

E

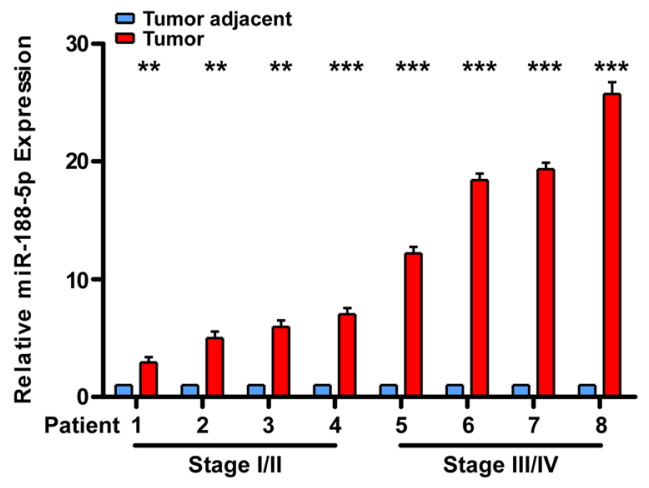

B

STAD patients from TCGA dataset (both $\mathbf{N}$ stage and OS available $n=307$ )
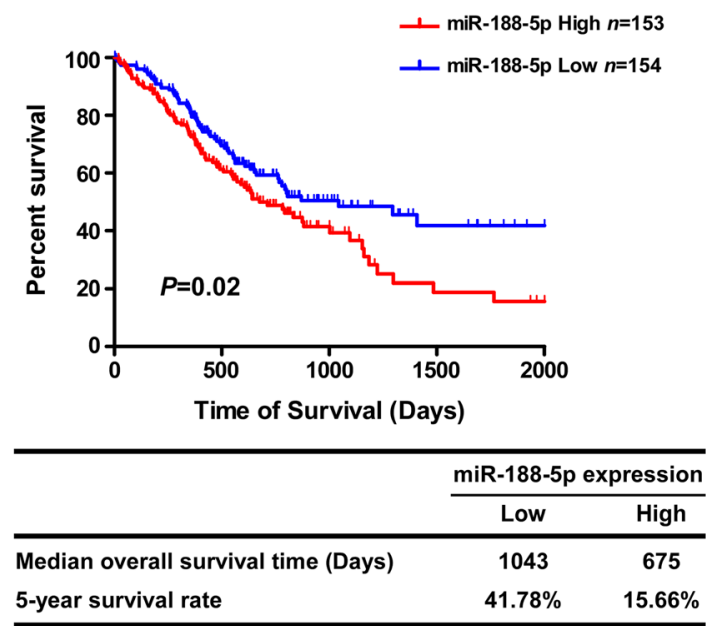

D

STAD patients from FMU cohort $(n=116)$
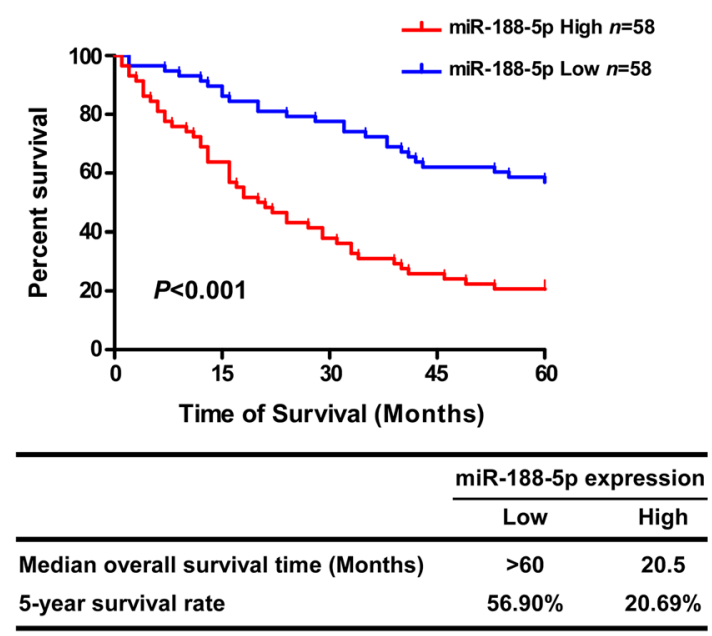

$\mathbf{F}$

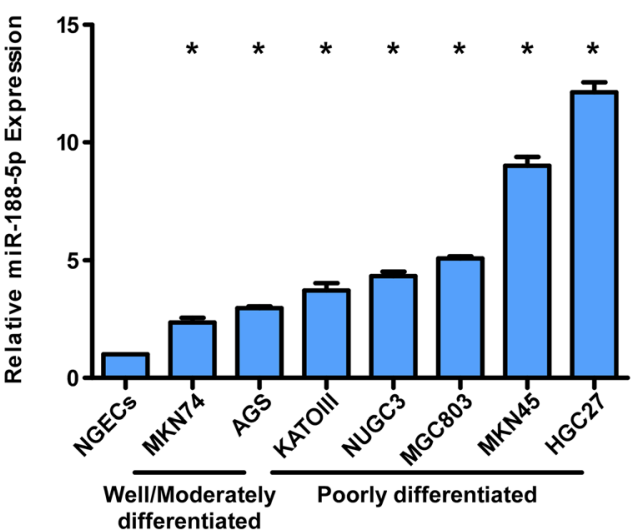

Fig. 1 (See legend on next page.) 


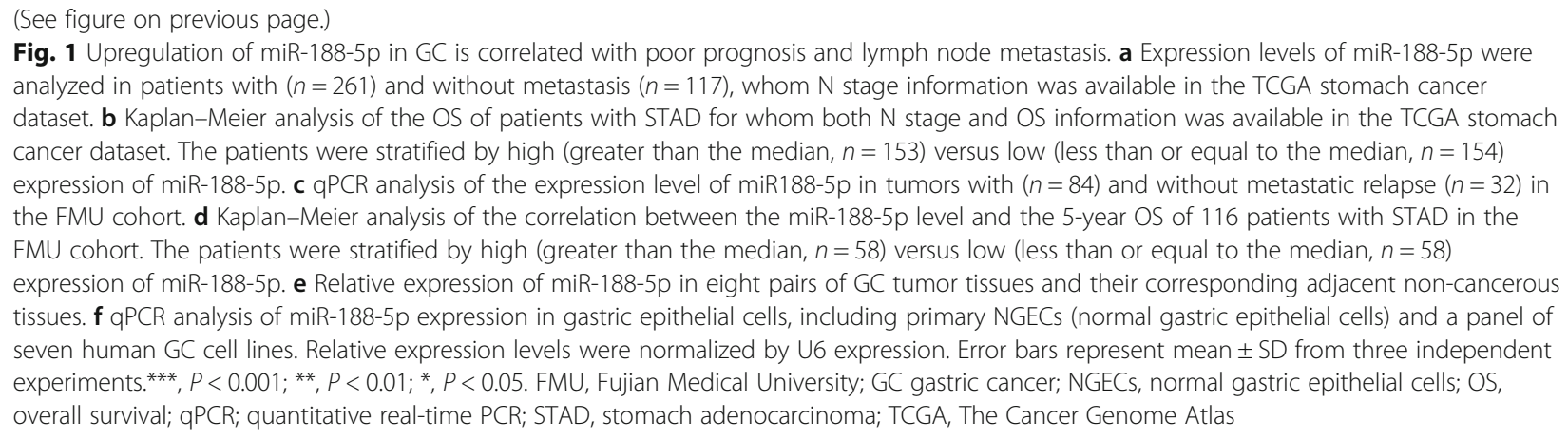

miR-188-5p promotes migration, invasion, and metastasis of GC cells

To elucidate the biological function of miR-188-5p in GC carcinogenesis, we transfected MGC803 (poorly differentiated) and AGS (moderately differentiated) cells with the miR-188-5p mimic or inhibitor. Overexpression of miR-188-5p did not alter the proliferation of MGC803 or AGS cells, as determined by CCK-8 assays (Additional file 7: Figure S2). However, GSEA of the TCGA STAD cohort showed that a higher level of miR188-5p was positively correlated with the gene signatures upregulated in metastatic cancer cells but inversely correlated with the gene signatures downregulated in metastasis cells (Fig. 2a) [22]. Furthermore, the migratory and invasive abilities of MGC803 and AGS cells were markedly enhanced by the miR-188-5p mimic but suppressed by the inhibitor, as determined by woundhealing/scratch assay (Fig. 2b) and Boyden chamber invasion assay (Fig. 2c).

The effect of miR-188-5p on metastatic potential in vivo was evaluated by generating the MGC803/miR-188$5 \mathrm{p}$ or AGS/miR-188-5p cells expressing luciferase and then injecting $2 \times 10^{6}$ cells into the caudal vain of BALB/ c nude mice. Additional file 8: Figure S3 showed that miR-188-5p expression was markedly increased in miR188-5p- overexpressing GC cells as compared with the control cells. Whole-mouse bioluminescence imaging was employed to serially monitor the appearance of metastases in living mice and the metastatic nodules on the lung surface of sacrificed mice were counted and detected by H\&E staining. Mice injected with MGC803 or AGS/miR-188-5p cells generated a significantly larger number of nodules in their lungs than those injected with the control cells (Fig. $2 \mathrm{~d}$ and e). Notably, all the five mice in the group injected with MGC803/miR188-5p cells died before 40 days after implantation while only three mice in the control group died by day 50 after inoculation (Fig. 2f, left). Likewise, AGS/ miR-188-5p injected mice survived for a shorter time than those injected with the control cells (Fig. 2f, right). Altogether, these results indicate that miR-188-
$5 \mathrm{p}$ functions to promote GC cells migration, invasion, and metastasis.

\section{miR-188-5p directly targets tumor suppressor PTEN}

To explore the underlying mechanism by which miR188-5p had the robust effect on GC metastasis, we first attempted to narrow down miR-188-5p target genes using TargetScan v6.2. The tumor suppressor gene, PTEN, was found to be suppressed by miR-188-5p (Fig. 3a). qPCR and western blot analysis found that both mRNA and protein levels of PTEN were drastically reduced in cells overexpressing miR-188-5p, but were elevated in cells transfected with the miR-188-5p-inhibitor (Fig. $3 \mathrm{~b}$ and c). Subsequently, the 3 '-UTR of PTEN was cloned into a luciferase reporter plasmid to evaluate whether miR-188-5p could bind to the PTEN $3{ }^{\prime}-\mathrm{UTR}$ leading to inhibition of luciferase reporter activity. As expected, a decrease in luciferase reporter activity in response to miR-188-5p expression was observed in MGC803 and AGS cells whereas miR-188-5p overexpression did not have inhibitory effects when the predicted miR-188-5p target/binding sites in the PTEN 3'UTR region were mutated (Fig. $3 \mathrm{~d}$ ). Therefore, these results strongly confirmed that miR-188-5p directly interacts with PTEN as a target.

To investigate the functional significance of miRNAmediated suppression of PTEN expression in the induction of cellular metastatic ability, we restored PTEN expression by transfecting a PTEN ectopic expression plasmid. As illustrated in Fig. 3e, PTEN overexpression inhibited the migratory and invasive capabilities of both the NC and miR-188-5p overexpressing GC cells in Transwell assays. Taken together, our data implicate that miR-188-5p promotes GC cell migration and invasion by suppressing PTEN.

\section{miR-188-5p promotes Wnt/ $\beta$-catenin signaling}

Since the Wnt/ $\beta$-catenin signaling pathway plays a crucial role in cancer metastasis we next examined whether miR-188-5p could impact Wnt/ $\beta$-catenin signaling activity. The results from GSEA analysis using the TCGA 
A

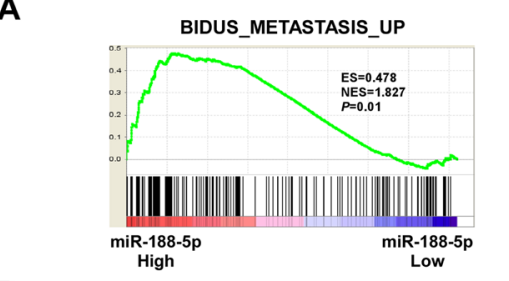

B

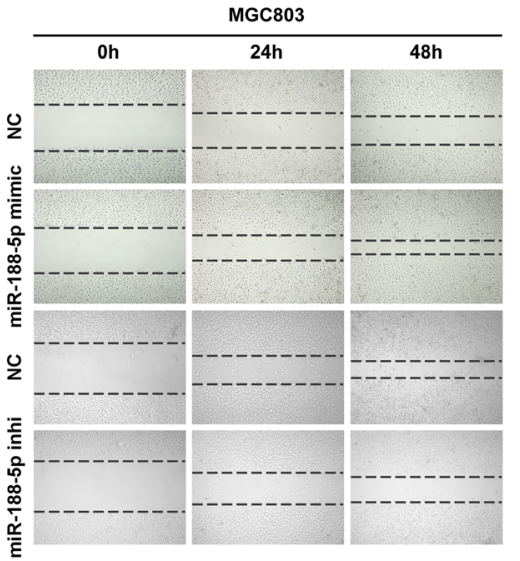

C

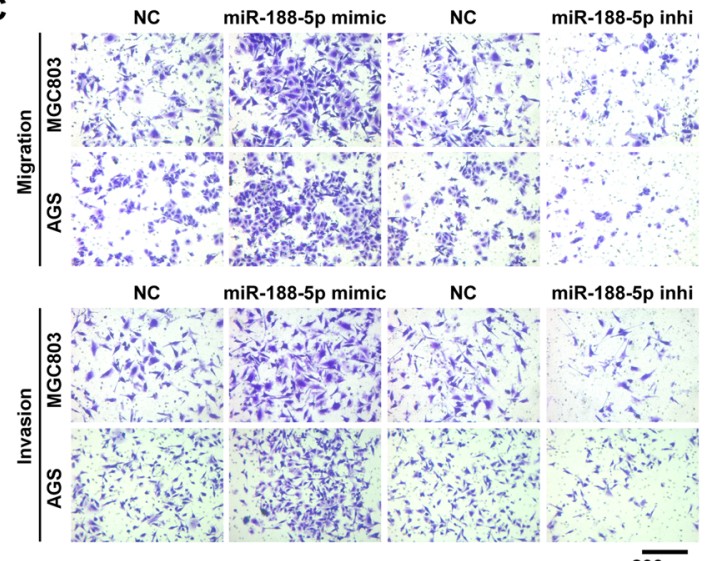

$2 \overline{200 \mu \mathrm{m}}$

D

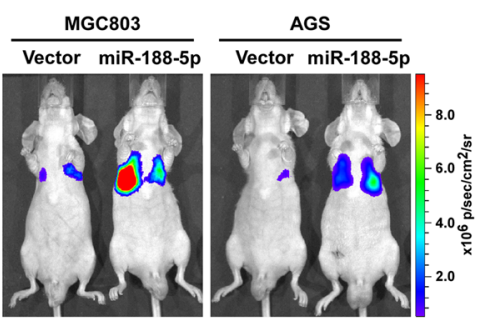

E
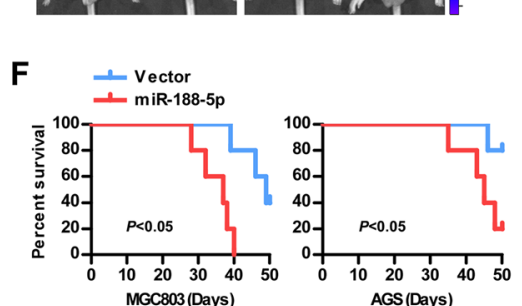
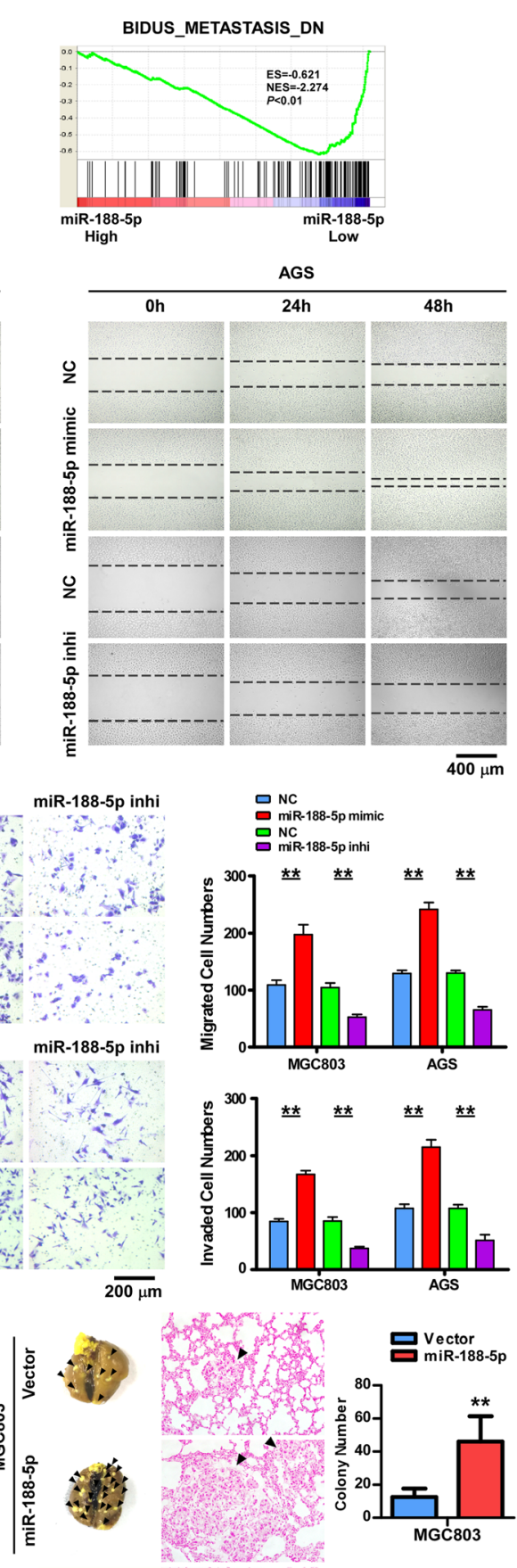

$\overline{400 \mu \mathrm{m}}$

$\therefore \mathrm{NC}$

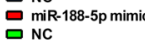
吕 NC
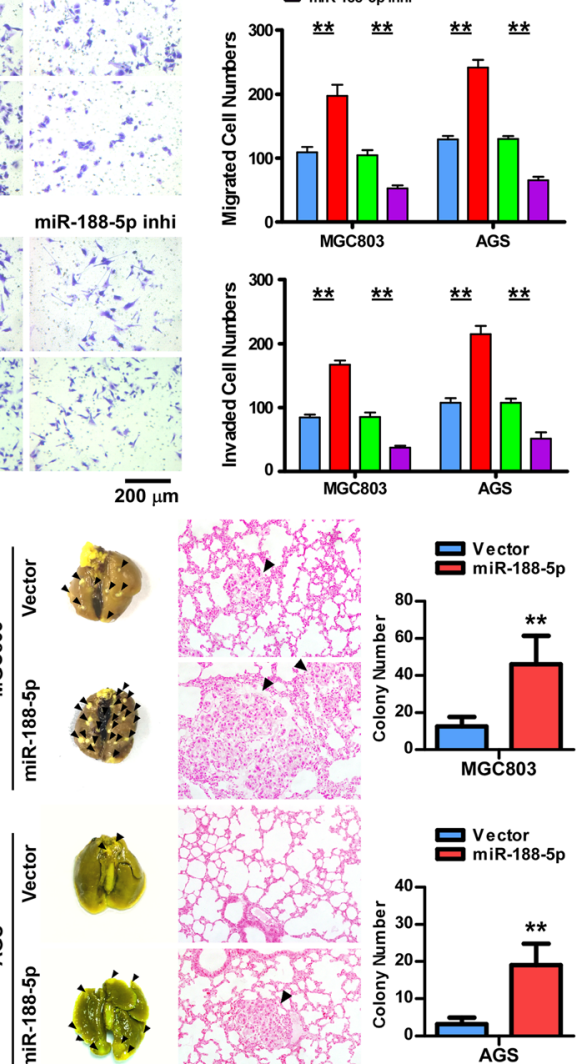

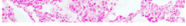

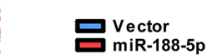

吕 $\begin{aligned} & \text { Vector } \\ & \text { miR-188-5p }\end{aligned}$

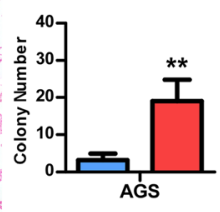

Fig. 2 (See legend on next page.) 
(See figure on previous page.)

Fig. 2 miR-188-5p promotes migration, invasion, and metastasis of GC cells. a GSEA plot showing miR-188-5p expression in association with metastasis-related gene signatures. The miR-188-5p level was positively correlated with activated metastasis-related gene signatures and inversely correlated with suppressed metastasis-related gene signatures in the TCGA stomach cancer dataset. b A wound-healing assay was performed with the indicated cells; Scale bar, $400 \mu \mathrm{m}$. c Representative images and quantification of Transwell assays in the indicated cells; Scale bar, $200 \mu \mathrm{m}$. d BALB/c nude mice injected intravenously via the caudal vein with indicated cells were monitored with a luciferase live-imaging system at indicated time points. Heat map scale bar represents photon emission. e Representative bright-field imaging of the lungs on 50 days of mice (left); H\&E staining of lung metastases formed by the indicated cells (middle) and number of visible surface metastatic lesions in mice ( $n=5$ per group) injected intravenously with indicated cells (right), Scale bar, $200 \mu \mathrm{m}$. Error bars represent mean \pm SD from three independent experiments. f Survival curves of mice injected intravenously with indicated cells. Error bars represent mean \pm SD ( $n=5$ per group). **, $P<0.01$. GC gastric cancer; GSEA, gene set enrichment analysis; H\&E, hematoxylin and eosin; NC, normal control; TCGA, The Cancer Genome Atlas

stomach cancer data set revealed that the miR-188-5p level was in a positive correlation with Wnt-activated gene signatures but an inverse correlation with Wntsuppressed gene signatures [23] (Fig. 4a and b), indicating that miR-188-5p might participate in $\mathrm{Wnt} / \beta$-catenin signaling activation. To address this issue, Top/Fop Flash reporter assays were used to evaluate the effects of miR188-5p on the $\beta$-catenin transcriptional activity in GC Fig. 4c showed that ectopic expression of miR-188-5p in MGC803 and AGS cells could significantly increase the $\beta$-catenin transcriptional activity whereas the miR-188-5p inhibitor demonstrated the opposite effects. Fluorescent microscopy was then utilized for detection of $\beta$-catenin intracellular localization. The results demonstrated that miR-188-5p overexpression in GC cells significantly enhanced $\beta$-catenin nuclear accumulation (Fig. $4 \mathrm{~d}$ ). We next examined the effects of miR-188-5p on the mRNA levels of the seven well-known effector genes of the Wnt/ $\beta$-catenin signaling. Expectedly, miR-188-5p markedly enhanced the expression of the target genes while the miR188-5p inhibitor reduced the expression (Fig. 4e).

Furthermore, we explored the role of Wnt/ $\beta$-catenin activation in miR-188-induced metastasis. As shown in Fig. 4f and g, knockdown of $\beta$-catenin or TCF4 inhibited $\beta$-catenin transcriptional activity and substantially reversed the migratory and invasive ability of miR-188-5poverexpressing GC cells, suggesting that miR-188-5p overexpression promote $\mathrm{GC}$ cells metastasis by activating $\mathrm{Wnt} / \beta$-catenin signaling.

\section{miR-188-5p activates $W n t / \beta$-catenin signaling via PTEN- Akt-GSK3 $\beta$ cascades}

To determine the role of PTEN in miR-188-5p mediated Wnt/ $\beta$-catenin signaling, we introduce PTEN-TSB to specifically inhibit the binding of miR-188-5p to PTEN mRNA in the control vector transfected and miR-188-5p overexpressing GC cells. The results showed that PTENTSB not only abrogated the inhibitory effects of miR188-5p on the 3' UTR of PTEN (Fig. 5a) but also reversed the effect of miR-188-5p on promoting $\beta$-catenin transcriptional activity (Fig. 5b). These results strongly supported the notion that miR-188-5p could specifically inhibit PTEN and activates $\mathrm{Wnt} / \beta$-catenin signaling.
Crosstalk between the PI3K/Akt and Wnt/ $/$-catenin pathway has been established in several cancer types including GC [28-31]. In this crosstalk, PTEN phosphorylates and inactivates GSK3 $\beta$ via Akt, which results in $\beta$ catenin accumulation and shuttling to the nucleus, thereby increasing target gene transcription [32]. Subsequently, we assessed the status of the PI3k/Akt pathway in the context of miR-188-5p with PTEN TSB by examining the phosphorylation status of Akt and GSK3 $\beta$. As shown in Fig. 5c, upregulation of miR-188-5p in MGC803 (left) and AGS (right) cells markedly decreased PTEN expression and increasing of phosphorylation of Akt (Ser473) and GSK3 $\beta$ (Ser9) compared with vector cells (lane 1 and 3). However, PTEN TSB could almost entirely reverse the activation of PI3k/Akt pathway in GC cells initially enhanced by miR-188-5p (lane 3 and 4) . These results indicate that miR-188-5p enhances Wnt/ $\beta$-catenin activation through the PTEN-Akt- GSK3 $\beta$ signaling cascade.

\section{Clinical relevance of miR-188-5p and $\beta$-catenin activation in GC}

Finally, we evaluated whether miR-188-5p overexpression and its mediated activation of $\mathrm{Wnt} / \beta$-catenin were of clinical relevance in GC. Figure $6 \mathrm{a}$ and $\mathrm{b}$ showed that there was an inverse correlation between the expression of miR-188-5p and the expression of PTEN in the FMU cohort of $116 \mathrm{GC}$ clinical specimens. $55.2 \%$ of specimens with high miR-188-5p expression (32/58) showed low PTEN expression whereas $79.4 \%$ of specimens with low miR-188-5p expression (46/58) displayed high PTEN expression $\left(X^{2}\right.$-test, $\left.P<0.001\right)$. In particular, tumor specimens that expressed high levels of miR-188-5p also showed a more accumulation of nuclear $\beta$-catenin (based on immunohistochemistry) as compared with those expressing lower levels of miR-188-5p (77.6\% vs. 20.7\%). Consistently, the negative correlation between the expression of miR-188-5p and PTEN was also observed in the TCGA STAD cohort (Fig. 6c). Our data confirmed that miR-188-5p is negatively correlated with PTEN expression and positively correlated with nuclear $\beta$-catenin level. 


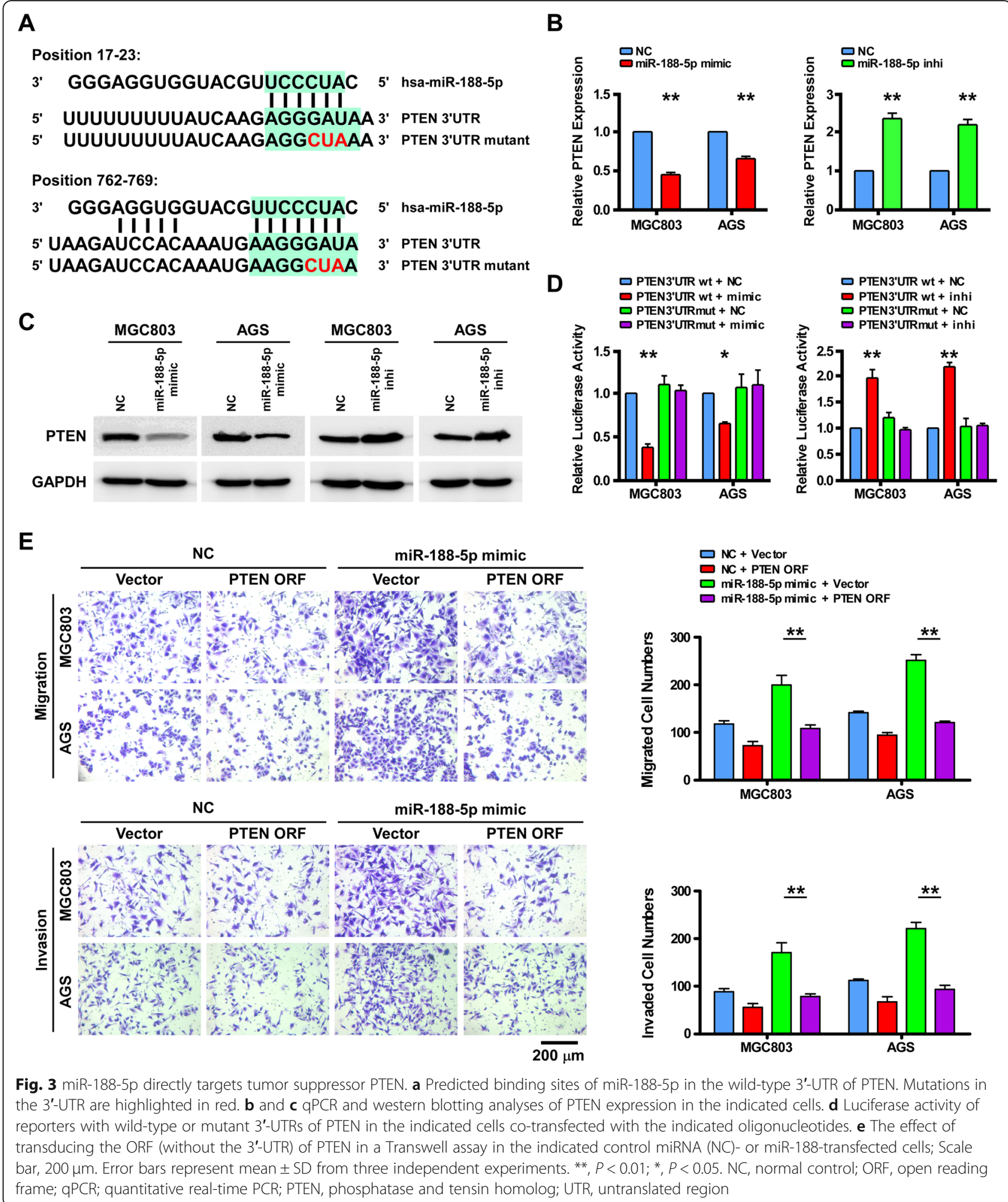

\section{Discussion}

Abnormal expression of miRNAs correlates with the tumorigenesis and progression of many human malignacies [14-16]. In the present study, the miRNA expression profiles of patients with STAD in the stomach cancer data set of TCGA were analyzed, and miR-188-5p was identified as the sole miRNA significantly correlated with both OS and lymph node metastasis status. This observation 
A

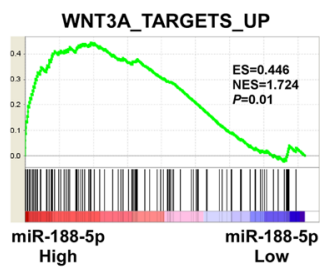

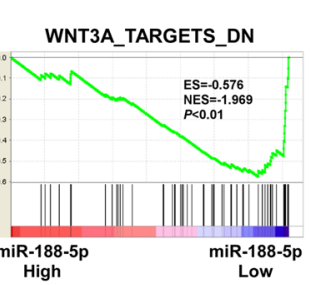
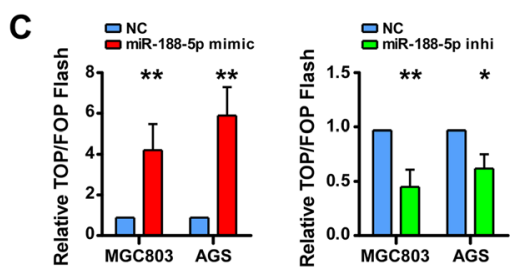

B

WNT3A_TARGETS_UP
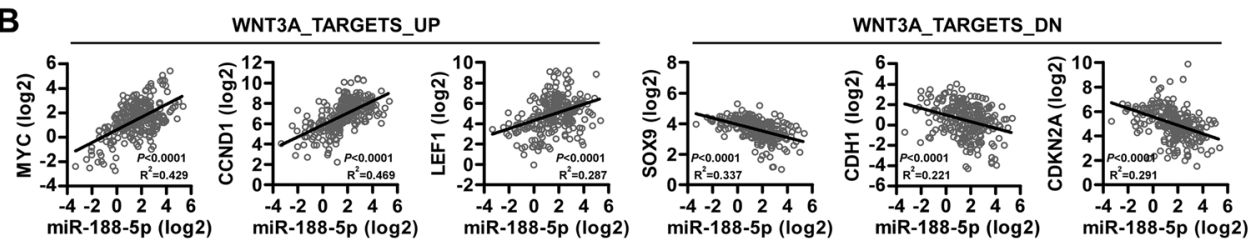

D
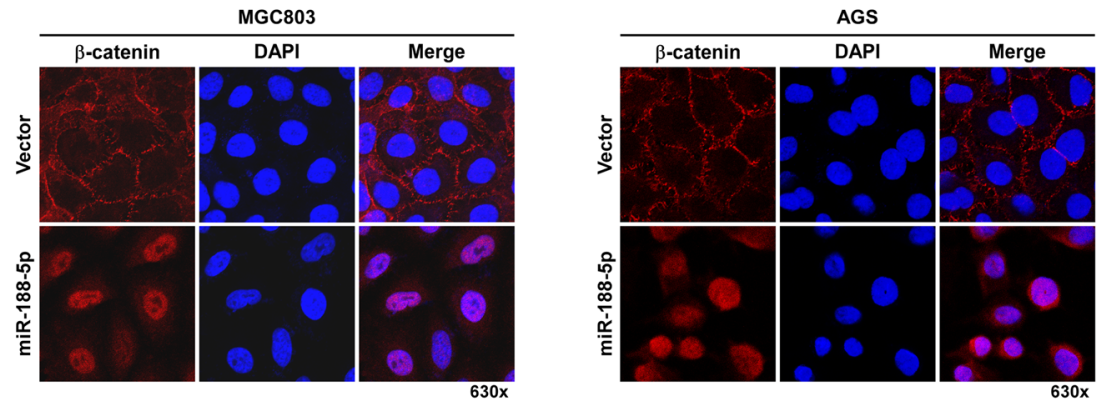

E $M G C 803-N C$

DGG803 - miR-188-5p mimic

DAGS - NC
AGS - miR-188-5p mimic

$\square$ MGC803 - NC

MGC803 - miR-188-5p inhi

AGS - NC
AGS - miR-188-5p inhi

F Vector + siControl

miR-188-5p + siControl
miR-188-5p + $\beta$-catenin-siRNA

$\underset{\text { MYC CCND1 TWIST MMP7 BMP4 FGF18 CD44 }}{\frac{\infty}{0}}$

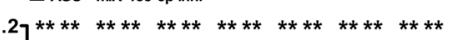

miR-188-5p + TCF4-siRNA
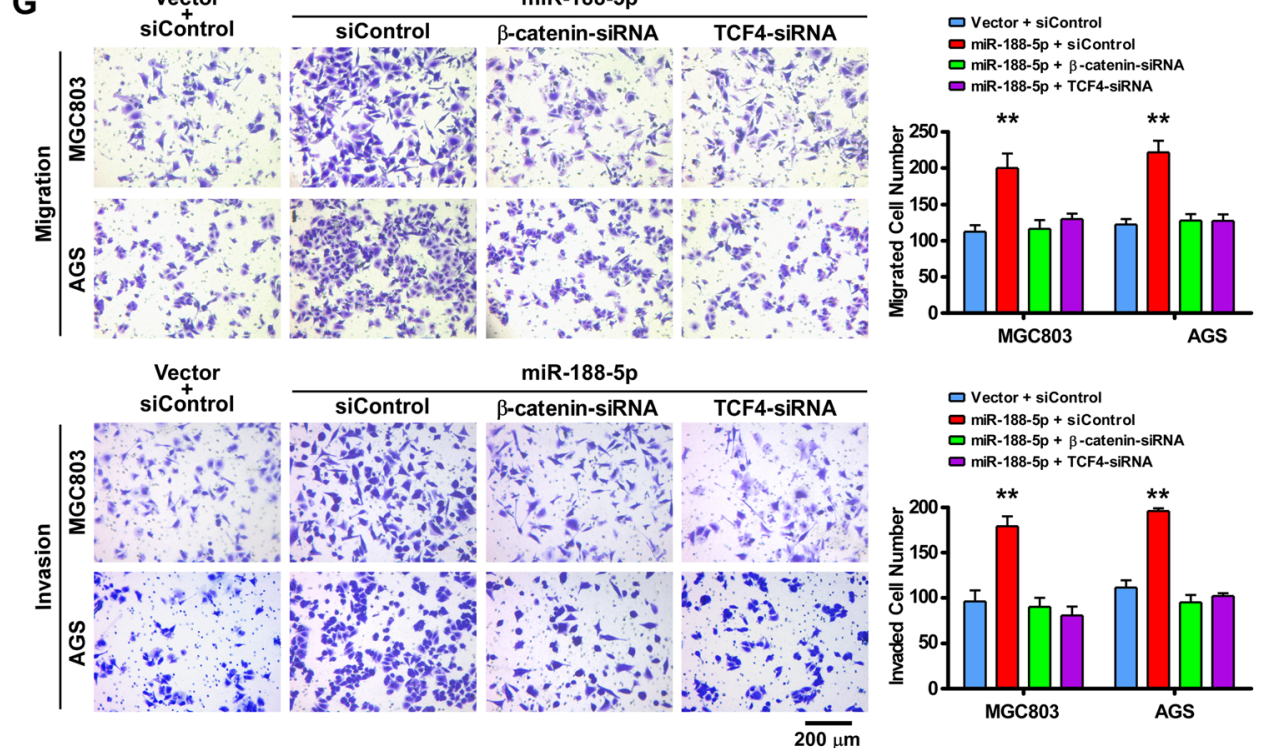

D Vector + siControl

- miR-188-5p + siControl

miR-188-5p + TCF4-siRNA

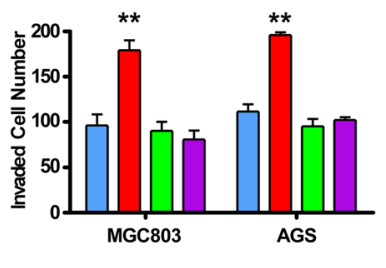

Fig. 4 (See legend on next page.) 
(See figure on previous page.)

Fig. 4 miR-188-5p promotes Wnt/B-catenin signaling. a GSEA plot showing that miR-188-5p expression was positively correlated with Wntactivated gene signatures and inversely correlated with Wnt-suppressed gene signatures in the TCGA stomach cancer dataset. b A linear regression analysis on the expression of Wnt pathway genes (the representative 3 upregulated and 3 downregulated Wnt target genes) versus miR-188-5p in the TCGA stomach cancer dataset. c The indicated cells were transfected with TOP or FOP reporter and Renilla pRL-TK plasmids and subjected to dual-luciferase assays $48 \mathrm{~h}$ after transfection. The detected reporter activity was normalized to the Renilla activity. $\mathbf{d}$ Subcellular $\beta$-catenin localization in the indicated cells was assessed by immunofluorescence staining; 630x. e qPCR analysis of the expression of the established downstream targets for the Wnt/ $\beta$-catenin pathway, including MYC, CCND1, TWIST, MMP7, BMP4, FGF18, and CD44, in the indicated cells. $\mathbf{f}$ Assay of TOP/FOP luciferase activity in the indicated cells. $\mathbf{g}$ Representative images of a Transwell assay in the indicated cells (left) and quantification of Transwell assay in the indicated cells (right). Scale bar, $200 \mu \mathrm{m}$. Error bars represent mean \pm SD from three independent experiments.**, $P<0.01$; ${ }^{*}, P<0.05$. GSEA, gene set enrichment analysis; NC, normal control; qPCR; quantitative real-time PCR; siRNA, short interfering RNA; TCGA, The Cancer Genome Atlas

was further confirmed in a set of 378 patients with STAD in the TCGA data and FMU cohort, which showed that miR-188-5p was highly upregulated in lymph node metastatic GC specimens compared with its level in nonlymph node metastatic samples. Furthermore, miR-188-5p was also significantly upregulated in M1 patients as compared with M0 patients in TCGA STAD cohort. These data suggested that miR-155-5p expression level is positively correlated with $\mathrm{GC}$ metastasis.

miR-188-5p was reported to function as a tumor suppressor in several cancers including non-small-cell lung cancer, glioma, hepatocellular carcinoma and prostate cancer [33-36]. In contrast, miR-188-5p is also shown to be an oncogenic miRNA that promotes prostate cancer cell proliferation and invasion, and take parts in aggressive progression and poor prognosis in patients with prostate cancer via inhibiting its target gene, ubiquitinconjugating enzyme E2I (UBE2I) [37]. In concert with this, in the present study we demonstrated that upregulation of miR-188-5p is involved in GC metastasis and poor patient survival. It is noteworthy that in a recent study, Peng et al. reported that miR-188-5p exerts tumor suppressor function in GC [38], which appears to disagree with our findings. The reasons for this discrepancy might be explained below. The TCGA data set and our FMU cohort used in the statistical analysis contained 378 and 116 GC samples respectively, which is greater than the sample number in Peng's clinical cohort $(n=$ 51). Using those much larger cohorts, we demonstrated that high miR-188-5p expression was significantly associated with poor survival of the GC patient and lymph node metastasis. In addition, it may also be notable that clinical information regarding the enrolled patients, pathological features and clinical stages was not available in Peng's paper for an invitation of the comparison.

Since chromosomal amplification/deletion or epigenetic modification changes are important upstream mechanisms of abnormal miRNA expression, we went further to analyze copy-number alterations (CNAs) and DNA methylation profiles from TCGA STAD data set. We found that both the copy number and DNA methylation level at miR188 locus were barely altered in STAD samples with the frequency of alteration being less than $1 \%$. In addition, we also employed the tool of Evolutionary Conserved Regions (ECR) Browser in attempt to identify distant gene regulatory elements and to predict transcription factor binding sites of miR188. We found that several signal transducer and activator of transcription (STAT) binding sites were enriched in the region of approximately $800 \mathrm{bp}$ upstream of miR188 locus. Functional experimental validations to confirm a binding relationship and regulatory effects are needed in the future.

To examine the influence of miR-188-5p on the development and progression of GC, miR-188-5p was overexpressed or inhibited in GC cell lines AGS and MGC803. The results revealed that the migratory and invasive abilities of MGC803 and AGS cells were markedly enhanced by the miR-188-5p mimic, but were suppressed by the inhibitor. Consistently, an in vivo metastasis experiment also confirmed that miR-188-5p significantly increased the pulmonary colonization of metastatic tumors. These data pinpoint a crucial role of miR-188-5p that plays as a tumor promoter in GC cell invasion and metastasis.

MiRNAs can negatively regulate their target genes via binding to the 3'-UTR of their mRNA to induce cleavage of the mRNAs or inhibit their translation [14]. It has reported that miR-188-5p plays distinct roles in different type of cancers by directly targeting multiple genes in a variety of scenarios as such MMP-2/13, MAP3K3, ZFP91, UBE2I, $\beta$-catenin, FGF5, LAPTM4B, Cyclins and CDKs in non-small-cell lung cancer, gastric cancer, glioma, hepatocellular carcinoma, prostate cancer or nasopharyngeal carcinoma [33-40]. However, the precise role of miR-188-5p and the underlying molecular mechanism in gastric cancer remains to be defined. In the present study, prediction of the candidate target genes for miR-188-5p was made using bioinformatic methods combined with luciferase reporter assays, qPCR, and western blot analysis. We showed that miR-188-5p could bind directly to the 3 '-UTR of PTEN and miR-188-5p overexpression downregulated PTEN expression at both the mRNA and protein levels. Moreover, restored expression of PTEN suppressed the migratory and invasive capabilities of both the NC and miR-188-5p overexpressing 
A

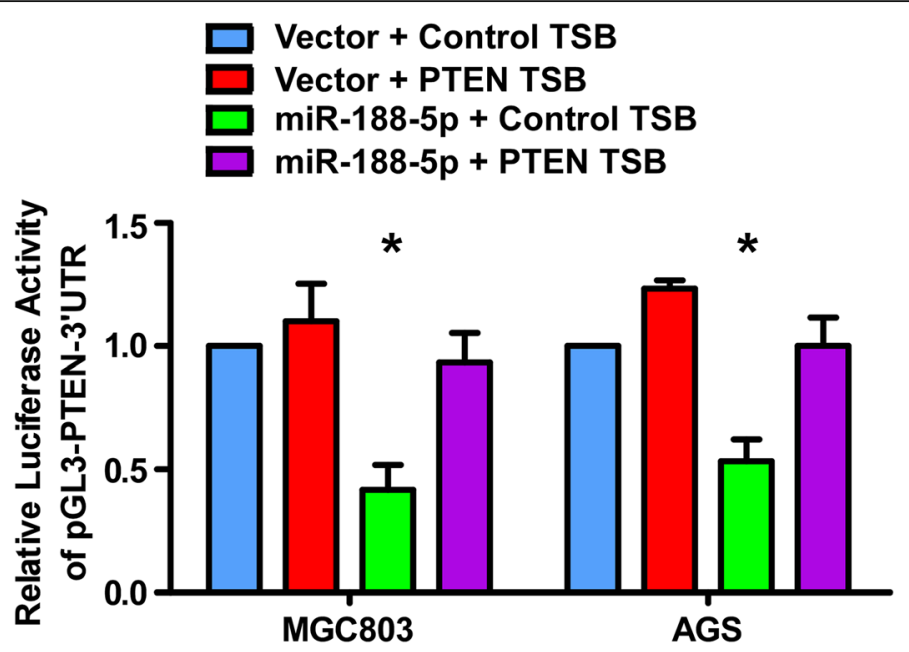

B

$\square$ Vector + Control TSB

$\square$ Vector + PTEN TSB

$\square$ miR-188-5p + Control TSB

$\square$ miR-188-5p + PTEN TSB

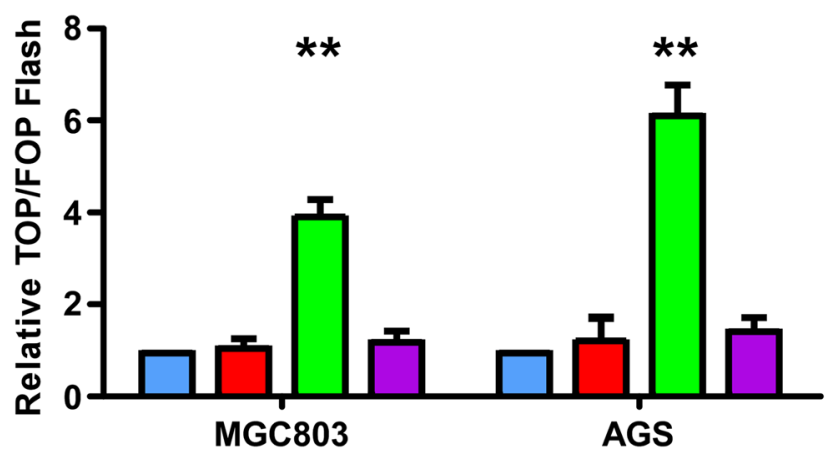

C

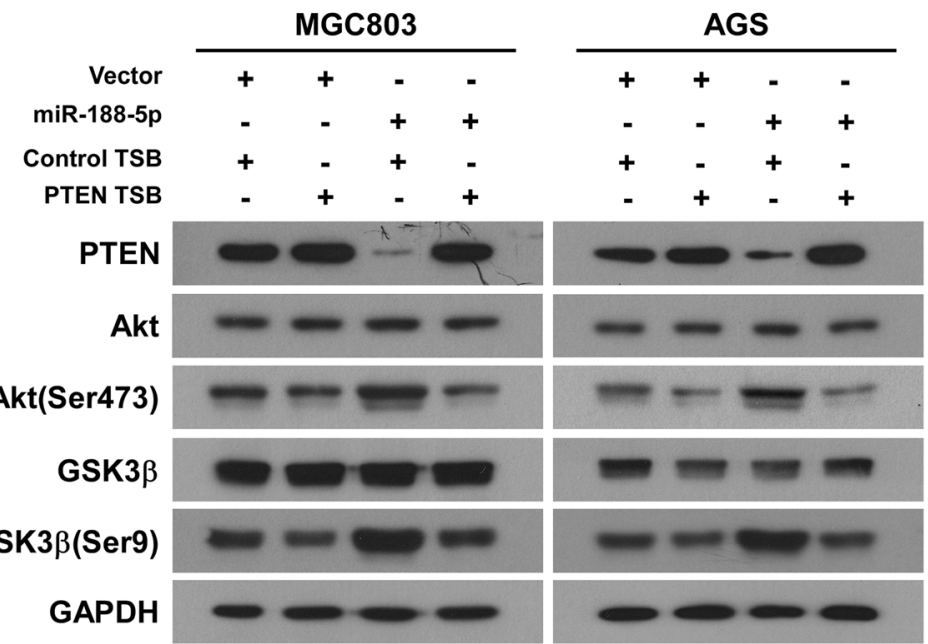

Fig. 5 (See legend on next page.) 
(See figure on previous page.)

Fig. 5 miR-188-5p activates Wnt/ $\beta$-catenin signaling through PTEN-Akt-GSK3 $\beta$ cascades. a Luciferase assay of the 3' UTR activity of PTEN in the indicated cells transfected with the indicated TSB. $\mathbf{b}$ Assay of TOP/FOP luciferase activity in the indicated cells. $\mathbf{c}$ Western blotting analyses of PTEN, Akt, p-Akt (Ser473), GSK3 $\beta$ and p-GSK3 $\beta$ (Ser9) expression in the indicated cells. Error bars represent mean \pm SD from three independent experiments. ${ }^{* *}, P<0.01 ;{ }^{*}, P<0.05$. NC, normal control; qPCR; quantitative real-time PCR; PTEN, phosphatase and tensin homolog; TSB, target site blocker; UTR, untranslated region

GC cells. Intriguingly, in a recent study of diabetic kidney disease (DKD) miR-188-5p was found to enhance renal tubular epithelial-mesenchymal transition (EMT) by a direct interaction with the PTEN 3'-untranslated region to suppress PTEN expression [41]. These results clearly indicate that miR-188-5p promotes the migration and invasion of GC cells, at least partially by suppressing PTEN.

PTEN is a well-known tumor suppressor, yet often inactivated in many types of human cancers [42, 43]. It not only involves in the regulation of apoptosis, cell cycle and angiogenesis but also plays an essential role in suppression of tumor metastasis [42, 44]. Notably, the PTEN/Akt axis regulates tumor metastasis via $\mathrm{Wnt} / \beta$ - catenin signaling [45]. Loss of PTEN function may cause the activation of $W n t / \beta$-catenin signaling in cancers and is also associated with tumor invasion and metastasis, implying that the pro-metastatic effect of PTEN deficiency may be mediated, at least partially, by the activation of $\beta$-catenin [12]. Consistently, we also found that knockdown of PTEN resulted in both activation of $\beta$ catenin and promotion of cell invasion, further attesting to the concept that the $\beta$-catenin-mediated pro-invasive function due to PTEN loss might also contribute to miR-188-5p-induced metastasis in GC. In this context, our observation that PTEN-TSB significantly reversed the effect of miR-188-5p on activating Wnt/ $\beta$-catenin

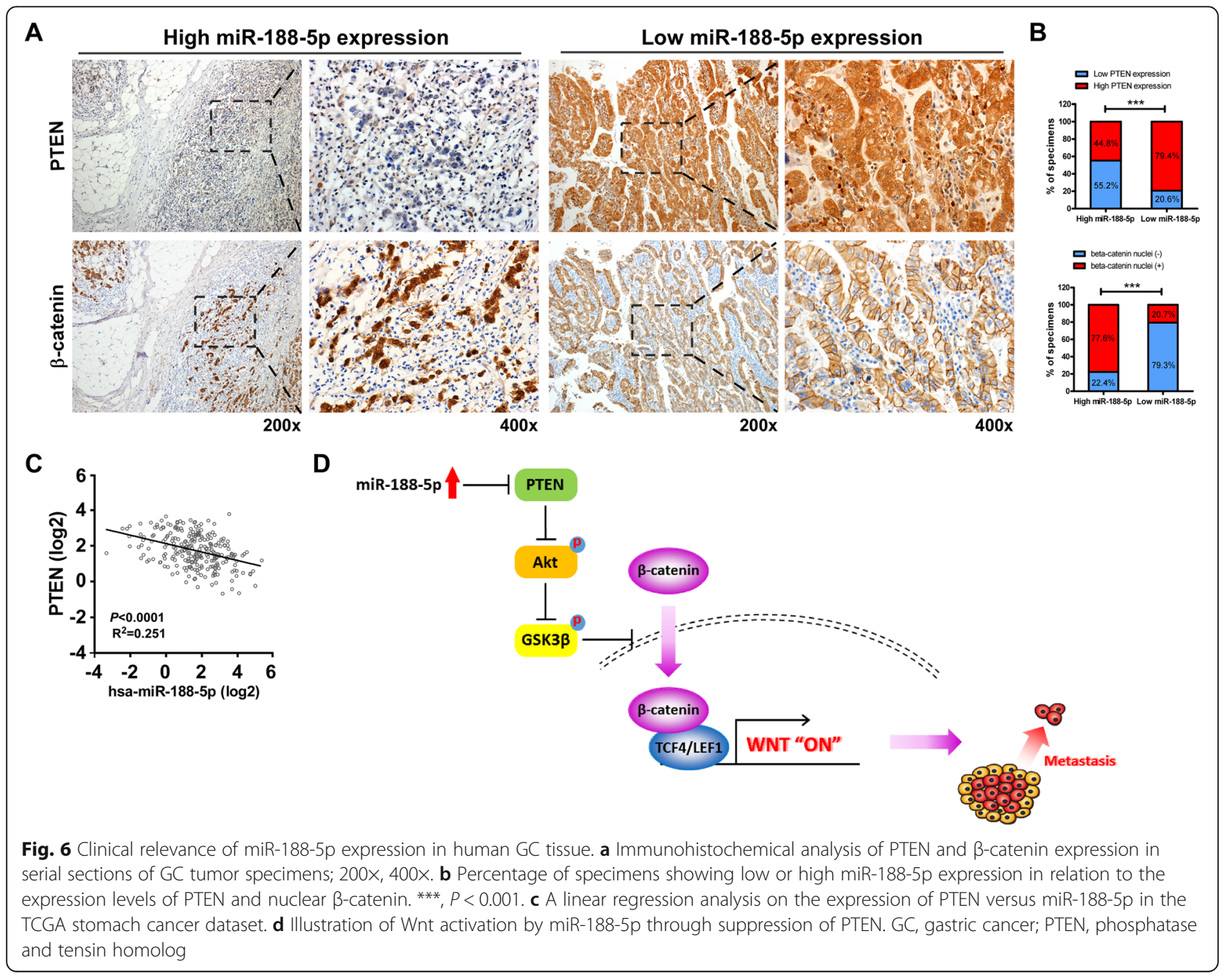


signaling in GC cell lines, suggested that suppression of PTEN is necessary for the pro-metastatic property of miR-188-5p upregulation.

\section{Conclusions}

In summary, our results demonstrated that miR-188-5p, by targeting PTEN, increases Akt and GSK3 $\beta$ phosphorylation, thus promoting $\beta$-catenin nuclear accumulation and activating Wnt/ $\beta$-catenin signaling, which enhances cancer metastasis and leads to poor prognosis in patients with GC (Fig. 6d). Exploration of the function and pathogenicity of miR-188-5p not only increased our understanding of GC carcinogenesis and progression but also identified miR-188-5p as a potential molecular biomarker and therapeutic target for the diagnosis and treatment of GC.

\section{Additional files}

Additional file 1: Excel spreadsheet 1. Accession codes of TCGA STAD samples. (XLSX $18 \mathrm{~kb})$

Additional file 2: Table S1. Clinicopathological characteristics of studied patients and expression of miR-188-5p in STAD from TCGA Dataset. (DOCX $16 \mathrm{~kb}$ )

Additional file 3: Table S2. Clinicopathological characteristics of studied patients and expression of miR-188-5p in STAD from the FMU Cohort. (DOCX $15 \mathrm{~kb}$ )

Additional file 4: Table S3. The sequences of primers used in plasmid construction. (DOCX $14 \mathrm{~kb}$ )

Additional file 5: Table S4. The sequences of primers used in real-time RT-PCR assay. (DOCX $14 \mathrm{~kb}$ )

Additional file 6: Figure S1. A. Expression of miR-188-5p and miR-188$3 p$ in human stomach cancer clinical specimens from the TCGA miRNA HiSeq expression array data. ${ }^{* *}, P<0.001$. B. Expression of miR-188-5p in different M (distant metastasis) classification of TCGA STAD cohort data. * $P<0.05$. C. Expression of miR-188-5p in human gastric cancer tissues and adjacent non-tumor tissues in TCGA STAD cohort data. $* * *, P<0.001$. D. Expression of miR-188-5p between human gastric cancer tissues and matched adjacent non-tumor tissues in TCGA STAD cohort data. Paired $t$ test, $P<0.001$. E. Increased fold of miR-188-5p expression between paired human gastric cancer tissues and matched adjacent non-tumor tissues samples shown in Figure S1D. (TIF $716 \mathrm{~kb}$ )

Additional file 7: Figure S2. Growth curves of the indicated cells as determined by MTT assay. Error bars represent mean \pm SD from 3 independent experiments. (TIF $265 \mathrm{~kb}$ )

Additional file 8: Figure S3. qPCR analysis of miR-188-5p expression in the miR-188-5p overexpressed cells or the control cells. Error bars represent mean \pm SD from 3 independent experiments. ${ }^{* *}, P<0.01$. (TIF $110 \mathrm{~kb}$ )

\section{Abbreviations}

Akt: AKT serine/threonine kinase; APC: Adenomatous polyposis coli; BMP4: Bone morphogenetic protein 4; CCK-8: The Cell Counting Kit-8; CCND1: Cyclin D1; CD44: CD44 molecule; CK1 a: Casein kinase 1 alpha; CST: Cell signaling technology; DAB: Diaminobenzidine; DAPI: 4'6-diamidino2-phenylindole; FDR: False discovery rate; FGF18: Fibroblast growth factor 18; FMU: Fujian Medical University; FZD: Frizzled; GAPDH: Glyceraldehyde-3phosphate dehydrogenase; GC: Gastric cancer; GSEA: The gene set enrichment analysis; GSK3B: Glycogen synthase kinase 3 beta; LEF: Lymphoid enhancer binding factor; LNA: Locked nucleic acid; LRP5/6: LDL receptorrelated protein 5 or 6; miR: MicroRNA; MMP7: Matrix metalloproteinase 7; MYC: MYC proto-oncogene; NC: Normal control; NGEC: Normal gastric epithelial cell; ORF: Open reading frame; OS: Overall survival; PBS: Phosphate buffer solution; PCR: Polymerase chain reaction; PI3K: Phosphatidylinositol 3kinase; PTEN: Phosphatase and tensin homolog; qPCR: Quantitative real-time PCR; RT: Reverse transcription; SAM: The significance analysis of microarrays; Ser: Serine; SI: The staining index; STAD: Stomach adenocarcinoma; TCF: Transcription factor; TCGA: The cancer genome atlas; TSB: Target site blocker; TWIST: Twist family bHLH transcription factor; UTR: Untranslated region

\section{Acknowledgments}

Not applicable.

\section{Authors' contributions}

$Y L$ and $X L Y$ conceived and designed the experiments, performed the experiments and analyzed the data and wrote the manuscript. YH collected and analyzed clinic samples. JX, LYL and JJS performed the experiments and analyzed the data. WNC, XJL and XL conceived and designed the study, and participated in its design and coordination and wrote the manuscript. All authors read and approved the final manuscript.

\section{Funding}

This work was supported by the National Natural Science Foundation of China [grant number 81502125], Foundation of Fujian Educational Committee [grant number JK2015016] and Medical Elite Cultivation Program of Fujian, P.R.C [grant number 2013-ZQN-ZD-24].

The funding bodies had no role in: the design of the study; data collection, analysis, or interpretation; or, in writing the manuscript.

\section{Availability of data and materials}

The datasets generated and/or analysed during the current study are available from the corresponding author on reasonable request. Prediction of miR-188-5p in association with metastasis-related and Wnt/ $\beta$-catenin signaling pathways was made based on the bioinformatics algorithms of gene set enrichment analysis (GSEA) through The Cancer Genome Atlas (TCGA) (http://tcga-data.nci.nih.gov/tcga).

\section{Ethics approval and consent to participate}

All patients provided their written informed consent in accordance with the Declaration of Helsinki and this study was approved by the institutional review board and regulatory authorities of Fujian Medical University (FMUIRB 16-078).

Animal studies were approved by the Fujian Medical University Institutional Animal Care and Use Committee (FMU-IACUC 15-066).

\section{Consent for publication}

Not applicable.

\section{Competing interests}

The authors declare that they have no competing interests.

\section{Author details}

${ }^{1}$ Key Laboratory of Gastrointestinal Cancer (Fujian Medical University), Ministry of Education, Fuzhou, China. ${ }^{2}$ Institute of Tissue Transplantation and Immunology and Department of Immunobiology, College of Life Science and Technology, Jinan University, Guangzhou, China. ${ }^{3}$ Fujian Key Laboratory of Tumor Microbiology, Fujian Medical University, Fuzhou, China.

Received: 24 October 2018 Accepted: 17 May 2019

Published online: 28 May 2019

\section{References}

1. Uemura N, Okamoto S, Yamamoto S, Matsumura N, Yamaguchi S, Yamakido M, Taniyama K, Sasaki N, Schlemper RJ. Helicobacter pylori infection and the development of gastric cancer. N Engl J Med. 2001;345(11):784-9.

2. Oue N, Aung PP, Mitani Y, Kuniyasu H, Nakayama H, Yasui W. Genes involved in invasion and metastasis of gastric cancer identified by arraybased hybridization and serial analysis of gene expression. Oncology. 2005; 69(Suppl 1):17-22.

3. Kim B, Koo H, Yang S, Bang S, Jung Y, Kim Y, Kim J, Park J, Moon RT, Song K, et al. TC1(C8orf4) correlates with Wnt/beta-catenin target genes and aggressive biological behavior in gastric cancer. Clin Cancer Res. 2006;12(11 Pt 1):3541-8. 
4. Kurayoshi M, Oue N, Yamamoto H, Kishida M, Inoue A, Asahara T, Yasui W, Kikuchi A. Expression of Wnt-5a is correlated with aggressiveness of gastric cancer by stimulating cell migration and invasion. Cancer Res. 2006;66(21):10439-48.

5. Zhang N, Zhang J, Shuai L, Zha L, He M, Huang Z, Wang Z. Kruppel-like factor 4 negatively regulates beta-catenin expression and inhibits the proliferation, invasion and metastasis of gastric cancer. Int J Oncol. 2012; 40(6):2038-48

6. Bhanot $P$, Brink $M$, Samos $C H$, Hsieh JC, Wang Y, Macke JP, Andrew D, Nathans J, Nusse R. A new member of the frizzled family from Drosophila functions as a wingless receptor. Nature. 1996;382(6588):225-30.

7. Tamai K, Semenov M, Kato Y, Spokony R, Liu C, Katsuyama Y, Hess F, SaintJeannet JP, He X. LDL-receptor-related proteins in Wnt signal transduction. Nature. 2000;407(6803):530-5.

8. Clevers H, Nusse R. Wnt/beta-catenin signaling and disease. Cell. 2012; 149(6):1192-205

9. Beroud C, Soussi T. APC gene: database of germline and somatic mutations in human tumors and cell lines. Nucleic Acids Res. 1996;24(1):121-4.

10. Kishida S, Yamamoto H, Ikeda S, Kishida M, Sakamoto I, Koyama S, Kikuchi A. Axin, a negative regulator of the wnt signaling pathway, directly interacts with adenomatous polyposis coli and regulates the stabilization of betacatenin. J Biol Chem. 1998;273(18):10823-6.

11. Perry JM, He XC, Sugimura R, Grindley JC, Haug JS, Ding S, Li L. Cooperation between both Wnt/\{beta\}-catenin and PTEN/PI3K/Akt signaling promotes primitive hematopoietic stem cell self-renewal and expansion. Genes Dev. 2011;25(18):1928-42

12. Korkaya H, Paulson A, Charafe-Jauffret E, Ginestier C, Brown M, Dutcher J, Clouthier SG, Wicha MS. Regulation of mammary stem/progenitor cells by PTEN/Akt/beta-catenin signaling. PLoS Biol. 2009;7(6):e1000121.

13. Mulholland DJ, Dedhar S, Wu H, Nelson CC. PTEN and GSK3beta: key regulators of progression to androgen-independent prostate cancer. Oncogene. 2006;25(3):329-37.

14. Garzon R, Calin GA, Croce CM. MicroRNAs in Cancer. Annu Rev Med. 2009; 60:167-79.

15. Lu J, Getz G, Miska EA, Alvarez-Saavedra E, Lamb J, Peck D, Sweet-Cordero A, Ebert BL, Mak RH, Ferrando AA, et al. MicroRNA expression profiles classify human cancers. Nature. 2005:435(7043):834-8.

16. Volinia S, Calin GA, Liu CG, Ambs S, Cimmino A, Petrocca F, Visone R, lorio $M$, Roldo C, Ferracin $M$, et al. A microRNA expression signature of human solid tumors defines cancer gene targets. Proc Natl Acad Sci U S A. 2006; 103(7):2257-61.

17. Cai J, Guan H, Fang L, Yang Y, Zhu X, Yuan J, Wu J, Li M. MicroRNA-374a activates Wnt/beta-catenin signaling to promote breast cancer metastasis. J Clin Invest. 2013;123(2):566-79.

18. Xia H, Ooi LL, Hui KM. MiR-214 targets beta-catenin pathway to suppress invasion, stem-like traits and recurrence of human hepatocellular carcinoma. PLoS One. 2012;7(9):e44206.

19. Strillacci A, Valerii MC, Sansone P, Caggiano C, Sgromo A, Vittori L, Fiorentino M, Poggioli G, Rizzello F, Campieri M, et al. Loss of miR-101 expression promotes Wnt/beta-catenin signalling pathway activation and malignancy in colon cancer cells. J Pathol. 2013;229(3):379-89.

20. Simon R, Lam A, Li MC, Ngan M, Menenzes S, Zhao Y. Analysis of gene expression data using BRB-ArrayTools. Cancer Inform. 2007;3:11-7.

21. Tusher VG, Tibshirani R, Chu G. Significance analysis of microarrays applied to the ionizing radiation response. Proc Natl Acad Sci U S A. 2001;98(9):5116-21.

22. Bidus MA, Risinger Jl, Chandramouli GV, Dainty LA, Litzi TJ, Berchuck A, Barrett JC, Maxwell GL. Prediction of lymph node metastasis in patients with endometrioid endometrial cancer using expression microarray. Clin Cancer Res. 2006;12(1):83-8

23. Labbe E, Lock L, Letamendia A, Gorska AE, Gryfe R, Gallinger S, Moses HL, Attisano L. Transcriptional cooperation between the transforming growth factor-beta and Wnt pathways in mammary and intestinal tumorigenesis. Cancer Res. 2007;67(1):75-84.

24. Subramanian A, Tamayo P, Mootha VK, Mukherjee S, Ebert BL, Gillette MA, Paulovich A, Pomeroy SL, Golub TR, Lander ES, et al. Gene set enrichment analysis: a knowledge-based approach for interpreting genome-wide expression profiles. Proc Natl Acad Sci U S A. 2005;102(43):15545-50.

25. Zheng $H$, Zhang F, Lin X, Huang C, Zhang Y, Li Y, Lin J, Chen W, Lin X. MicroRNA-1225-5p inhibits proliferation and metastasis of gastric carcinoma through repressing insulin receptor substrate- 1 and activation of betacatenin signaling. Oncotarget. 2016;7(4):4647-63.

26. Liu K, Li G, Fan C, Diao Y, Wu B, Li J. Increased expression of MicroRNA-221 in gastric cancer and its clinical significance. J Int Med Res. 2012;40(2):467-74.
27. Loffek S, Schilling O, Franzke CW. Series "matrix metalloproteinases in lung health and disease": biological role of matrix metalloproteinases: a critical balance. Eur Respir J. 2011;38(1):191-208.

28. Nakayama M, Hisatsune J, Yamasaki E, Isomoto H, Kurazono H, Hatakeyama M, Azuma T, Yamaoka Y, Yahiro K, Moss J, et al. Helicobacter pylori VacAinduced inhibition of GSK3 through the PI3K/Akt signaling pathway. J Biol Chem. 2009;284(3):1612-9.

29. Sokolova O, Bozko PM, Naumann M. Helicobacter pylori suppresses glycogen synthase kinase 3 beta to promote beta-catenin activity. J Biol Chem. 2008;283(43):29367-74.

30. Sharma M, Chuang WW, Sun Z. Phosphatidylinositol 3-kinase/Akt stimulates androgen pathway through GSK3beta inhibition and nuclear beta-catenin accumulation. J Biol Chem. 2002;277(34):30935-41.

31. Agarwal A, Das K, Lerner N, Sathe S, Cicek M, Casey G, Sizemore N. The AKT/ I kappa B kinase pathway promotes angiogenic/metastatic gene expression in colorectal cancer by activating nuclear factor-kappa B and beta-catenin. Oncogene. 2005;24(6):1021-31.

32. Monick MM, Mallampalli RK, Carter AB, Flaherty DM, McCoy D, Robeff PK, Peterson MW, Hunninghake GW. Ceramide regulates lipopolysaccharideinduced phosphatidylinositol 3-kinase and Akt activity in human alveolar macrophages. J Immunol. 2001;167(10):5977-85.

33. Zhao L, Ni X, Zhao L, Zhang Y, Jin D, Yin W, Wang D, Zhang W. MiroRNA188 acts as tumor suppressor in non-small-cell lung Cancer by targeting MAP3K3. Mol Pharm. 2018;15(4):1682-9.

34. Li N, Shi H, Zhang L, Li X, Gao L, Zhang G, Shi Y, Guo S. miR-188 inhibits glioma cell proliferation and cell cycle progression through targeting betacatenin. Oncol Res. 2018;26(5):785-94.

35. Fang F, Chang RM, Yu L, Lei X, Xiao S, Yang H, Yang LY. MicroRNA-188-5p suppresses tumor cell proliferation and metastasis by directly targeting FGF5 in hepatocellular carcinoma. J Hepatol. 2015;63(4):874-85.

36. Zhang H, Qi S, Zhang T, Wang A, Liu R, Guo J, Wang Y, Xu Y. miR-188-5p inhibits tumour growth and metastasis in prostate cancer by repressing LAPTM4B expression. Oncotarget. 2015;6(8):6092-104.

37. Zhang Y, Jiang F, He H, Ye J, Mao X, Guo Q, Wu SL, Zhong W, Wu CL, Lin N. Identification of a novel microRNA-mRNA regulatory biomodule in human prostate cancer. Cell Death Dis. 2018;9(3):301.

38. Peng Y, Shen $X$, Jiang H, Chen Z, Wu J, Zhu Y, Zhou Y, Li J. MiR-188-5p suppresses gastric cancer cell proliferation and invasion via targeting ZFP91. Oncol Res. 2018; 27(1):65-71.

39. Hou H, Gao F, Liang H, LV Y, Li M, Yao L, Zhang J, Dou G, Wang Y. MicroRNA-188-5p regulates contribution of bone marrow-derived cells to choroidal neovascularization development by targeting MMP-2/13. Exp Eye Res. 2018;175:115-23.

40. Wu J, Lv Q, He J, Zhang H, Mei X, Cui K, Huang N, Xie W, Xu N, Zhang Y. MicroRNA-188 suppresses G1/S transition by targeting multiple cyclin/CDK complexes. Cell Commun Signal. 2014;12:66

41. Xue M, Cheng Y, Han F, Chang Y, Yang Y, Li X, Chen L, Lu Y, Sun B, Chen L. Triptolide attenuates renal tubular epithelial-mesenchymal transition via the MiR-188-5p-mediated PI3K VAKT pathway in diabetic kidney disease. Int J Biol Sci. 2018;14(11):1545-57.

42. Mulholland DJ, Kobayashi N, Ruscetti M, Zhi A, Tran LM, Huang J, Gleave M, Wu H. Pten loss and RAS/MAPK activation cooperate to promote EMT and metastasis initiated from prostate cancer stem/progenitor cells. Cancer Res. 2012;72(7):1878-89.

43. Li J, Yen C, Liaw D, Podsypanina K, Bose S, Wang SI, PuC J, Miliaresis C, Rodgers L, McCombie R, et al. PTEN, a putative protein tyrosine phosphatase gene mutated in human brain, breast, and prostate cancer. Science. 1997;275(5308):1943-7.

44. Wikman H, Lamszus K, Detels N, Uslar L, Wrage M, Benner C, Hohensee I, Ylstra B, Eylmann K, Zapatka M, et al. Relevance of PTEN loss in brain metastasis formation in breast cancer patients. Breast Cancer Res. 2012;14(2):R49.

45. Wang H, Quah SY, Dong JM, Manser E, Tang JP, Zeng Q. PRL-3 downregulates PTEN expression and signals through PI3K to promote epithelialmesenchymal transition. Cancer Res. 2007;67(7):2922-6.

\section{Publisher's Note}

Springer Nature remains neutral with regard to jurisdictional claims in published maps and institutional affiliations. 